\title{
SOLAR SYSTEM MOONS AS ANALOGS FOR COMPACT EXOPLANETARY SYSTEMS
}

\author{
Stephen R. KANE ${ }^{1,2}$, Natalie R. Hinkel ${ }^{1,2}$, and Sean N. Raymond 3,4 \\ ${ }^{1}$ Department of Physics \& Astronomy, San Francisco State University, 1600 Holloway Avenue, San Francisco, CA 94132, USA; skane@ipac.caltech.edu \\ 2 NASA Exoplanet Science Institute, Caltech, MS 100-22, 770 South Wilson Avenue, Pasadena, CA 91125, USA \\ ${ }^{3}$ CNRS, UMR 5804, Laboratoire d'Astrophysique de Bordeaux, 2 rue de l'Observatoire, BP 89, F-33271 Floirac Cedex, France \\ ${ }^{4}$ Observatoire Aquitain des Sciences de l'Univers, Université de Bordeaux, 2 rue de l'Observatoire, BP 89, F-33271 Floirac Cedex, France \\ Received 2013 April 3; accepted 2013 September 4; published 2013 October 8
}

\begin{abstract}
The field of exoplanetary science has experienced a recent surge of new systems that is largely due to the precision photometry provided by the Kepler mission. The latest discoveries have included compact planetary systems in which the orbits of the planets all lie relatively close to the host star, which presents interesting challenges in terms of formation and dynamical evolution. The compact exoplanetary systems are analogous to the moons orbiting the giant planets in our solar system, in terms of their relative sizes and semimajor axes. We present a study that quantifies the scaled sizes and separations of the solar system moons with respect to their hosts. We perform a similar study for a large sample of confirmed Kepler planets in multi-planet systems. We show that a comparison between the two samples leads to a similar correlation between their scaled sizes and separation distributions. The different gradients of the correlations may be indicative of differences in the formation and/or long-term dynamics of moon and planetary systems.
\end{abstract}

Key words: planetary systems - planets and satellites: formation

\section{INTRODUCTION}

The field of exoplanetary science is constantly expanding and diversifying. The number of confirmed planets now exceeds 800 , though the number of detected exoplanets may greatly exceed this due to the vast number of exoplanet candidates found by the Kepler mission (Borucki et al. 2011a, 2011b; Batalha et al. 2013), since most of the multi-planet systems from the Kepler candidate sample are exoplanets rather than false-positives (Lissauer et al. 2012). Moreover, the number of detected systems with multiple planets within a relatively small semimajor axis has increased from both radial velocity (RV) and transit methods.

The compact multi-planet systems are particularly interesting because their orbital structure and dynamical stability are a challenge to explain in terms of formation mechanisms. For example, the Kepler-11 system consists of six detected planets, all of which lie within $0.5 \mathrm{AU}$ of the host star (Lissauer et al. 2011a). The RV technique was used to detect the compact system HD 10180, which has seven known exoplanets (possibly nine according to Tuomi 2012), four of which have orbital periods less than that of Mercury (Lovis et al. 2011). There are indications of trends between the relative radii of planets within a given multi-planet system (Ciardi et al. 2013). A particular trait of compact multi-planet systems is that they bear a close resemblance to the system of moons harbored by the giant planets of our own solar system when the sizes are placed in units of the host, be that a star or a planet. This was briefly noted in the case of the Kepler- 42 system, which consists of a low-mass star with three exoplanets (Muirhead et al. 2012) and closely resembles the Galilean system of moons.

We present an analysis and comparison of both solar system moons and Kepler compact multi-planet systems. We perform this analysis by scaling the radii and semimajor axis of each body to the radius of the host: a giant planet in the case of the solar system and a Kepler star in the case of the Kepler systems. Section 2 describes the analysis of the moons in detail and our power-law fit to the data for the regular (largest) moons.
Section 3 repeats this analysis in the context of the Kepler multiplanet systems, with application to the systems Kepler-9, 11, 18, $20,30,32,33$, and 42 . In Section 4 we quantify the statistical differences between the two populations. Section 5 presents calculations of the tidal dissipation timescales for the moons and planets as an additional diagnostic for comparison. Section 6 describes the comparison of the two samples and suggests implications for formation mechanisms and subsequent dynamical evolution in these environments. In particular, we show that variations in resonance-trapping mechanisms resulting from the relative disk masses and compositions are possible causes of differences in correlation gradients between the two samples. In Section 7, we briefly comment on how these results could be extended to brown dwarfs.

\section{MOONS WITHIN OUR SYSTEM}

Here we discuss the radius and semimajor axis distributions for known moons within our solar system. Moons are separated into the two broad categories of regular and irregular satellites. The former are generally thought to have formed out of the same protoplanetary disk that formed the planet. The latter are likely captured smaller bodies, which tend to have inclined and/or retrograde orbits.

We include data for 67, 62, 27, and 13 moons for Jupiter, Saturn, Uranus, and Neptune, respectively. These data were acquired from The Giant Planet Satellite and Moon Page, ${ }^{5}$ which is curated by Scott Sheppard (Sheppard \& Jewitt 2003; Sheppard et al. 2005, 2006). For each of the 169 solar system moons included in this study, we have scaled both the radii and moon-planet separation to the radius of the host planet. The resulting scaled radii and semimajor axes for the moons of all four giant planets are shown in the left panel of Figure 1.

It is striking in the left panel of Figure 1 how the moons follow the same distribution for all four of the giant planets. For radii less than 0.01 of the planet radius, the distribution becomes dominated by irregular satellites with a range of inclination

\footnotetext{
5 http://www.dtm.ciw.edu/users/sheppard/satellites/
} 

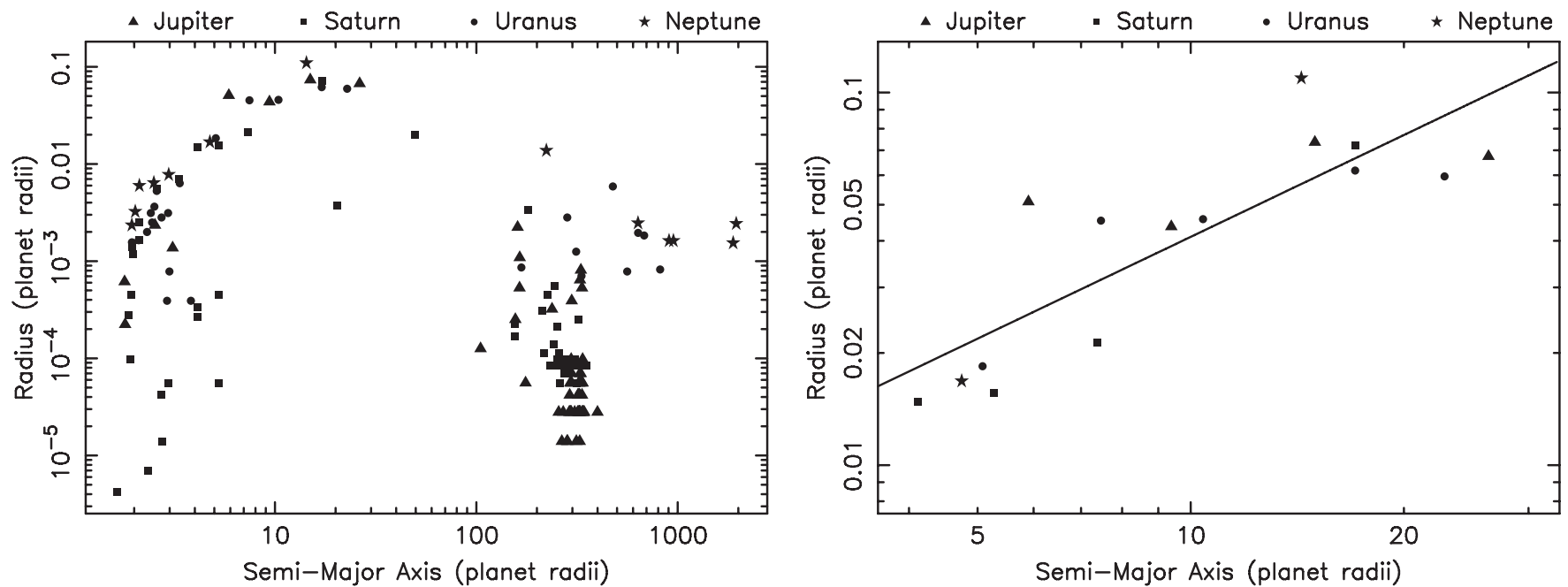

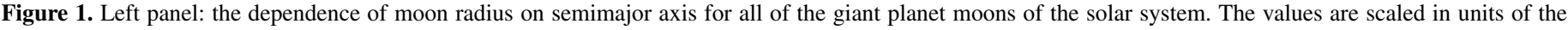
radius of the host planet. Right panel: zoomed in on the regular moons of the giant planets with a power-law fit to the data.

values and a mix of prograde and retrograde orbits (Frouard et al. 2011; Gaspar et al. 2011; Jewitt \& Haghighipour 2007). Those moons beyond 100 planetary radii are also dominated by irregular satellites whose large separations from the host planet are artifacts of their capture scenarios. The Neptunian moon system diverges from the pattern at the outer edge, likely due to interactions with and captures of Kuiper Belt Objects (Dawson \& Murray-Clay 2012; Levison et al. 2008). It has been suggested that Triton is a captured body, possibly from a Kuiper Belt Object binary (Agnor \& Hamilton 2006). The distribution of irregular satellites close to the planets is dominated by the shepherd moons of Saturn. The anomalous Saturn moon near the center of the plot is Hyperion, which is in 4:3 resonance with Titan (Colombo et al. 1974; Peale 1978).

The other major feature of this distribution is that exhibited by the large moons inside of 30 planet radii. There is a clear correlation of increasing radius with increasing moon-planet separation. The right panel of Figure 1 is a zoom of this region that shows the radius-separation trend more clearly. Table 1 contains the complete list of moons that fall within 30 planet radii, along with their absolute and scaled semimajor axes and radii. We fit a power law to these data of the form $y=c_{1} x^{c_{2}}$, where $c_{1}=0.00507, c_{2}=0.90777$, and $c_{2}$ describes the slope of the fit. The primary outlier from the fit to the data is the moon Triton which, as mentioned above, is likely a captured moon. In the next section, we perform a similar analysis for Kepler multi-planet systems to investigate the similarity of the correlation.

\section{COMPACT MULTI-PLANET SYSTEMS}

The Kepler mission has numerous multi-planet systems that have been confirmed using a combination of RV follow-up observations and transit timing variations. Here we use the published data for eight confirmed Kepler multi-planet systems that have three or more planets in the system: Kepler-9 (Holman et al. 2010; Torres et al. 2011), Kepler-11 (Lissauer et al. 2011a, 2013), Kepler-18 (Cochran et al. 2011), Kepler-20 (Fressin et al. 2012; Gautier et al. 2012), Kepler-30 (Sanchis-Ojeda et al. 2012), Kepler-32 (Fabrycky et al. 2012; Swift et al. 2013), Kepler-33 (Lissauer et al. 2012), and Kepler-42 (Muirhead et al. 2012). Using the confirmed multi-planet systems allows for a more robust analysis since the radii of the host stars are better
Table 1

Solar System Moon Separations and Radii

\begin{tabular}{lcccc}
\hline \hline Moon & $\begin{array}{c}a \\
(\mathrm{AU})\end{array}$ & $\begin{array}{c}R_{m} \\
\left(R_{\oplus}\right)\end{array}$ & $\begin{array}{c}a \\
(\text { Host Planet Radii) }\end{array}$ \\
& & & & \\
Jupiter & & & & $R_{m}$ \\
$\quad$ Io & 0.0028 & 0.572 & 5.900 & 0.051 \\
$\quad$ Europa & 0.0045 & 0.490 & 9.387 & 0.044 \\
$\quad$ Ganymede & 0.0072 & 0.826 & 14.972 & 0.074 \\
$\quad$ Callisto & 0.0126 & 0.757 & 26.334 & 0.067 \\
Saturn & & & & \\
$\quad$ Tethys & 0.0020 & 0.166 & 4.122 & 0.015 \\
$\quad$ Dione & 0.0025 & 0.175 & 5.279 & 0.016 \\
$\quad$ Rhea & 0.0035 & 0.240 & 7.373 & 0.021 \\
$\quad$ Titan & 0.0082 & 0.808 & 17.091 & 0.072 \\
Uranus & & & & \\
$\quad$ Miranda & 0.0009 & 0.074 & 5.082 & 0.018 \\
$\quad$ Ariel & 0.0013 & 0.182 & 7.469 & 0.045 \\
$\quad$ Umbriel & 0.0018 & 0.183 & 10.407 & 0.046 \\
Titania & 0.0029 & 0.248 & 17.070 & 0.062 \\
$\quad$ Oberon & 0.0039 & 0.239 & 22.830 & 0.060 \\
Neptune & & & & \\
$\quad$ Proteus & 0.0008 & 0.065 & 4.749 & 0.017 \\
$\quad$ Triton & 0.0024 & 0.425 & 14.327 & 0.109 \\
\hline
\end{tabular}

characterized than those for the Kepler candidates. We also impose a scaled semimajor axis cutoff of 120 host radii, beyond which the systems are no longer compact and comparisons with the solar system moons become less valid.

As for the solar system moons described in Section 2, we scale the radii and star-planet separations by the radius of the host star in each system, shown in Figure 2 with scaled radii uncertainties. The Kepler-20 system is specifically marked on the plot and is unusual because the two smaller planets are staggered between the three larger planets. Also, the relatively small planets in the Kepler-42 system have significantly larger radii uncertainties than those planets in the other systems of this sample. The absolute and scaled values for all of the Kepler planets included in this study are tabulated in Table 2.

The range of planet sizes included in our sample varies from $0.572 R_{\oplus}$ to $12.31 R_{\oplus}$. The radius distribution has a mean of $3.6 R_{\oplus}$ and a standard deviation of $2.9 R_{\oplus}$. Part of the reason for the relatively large scatter is the range of host star properties. 


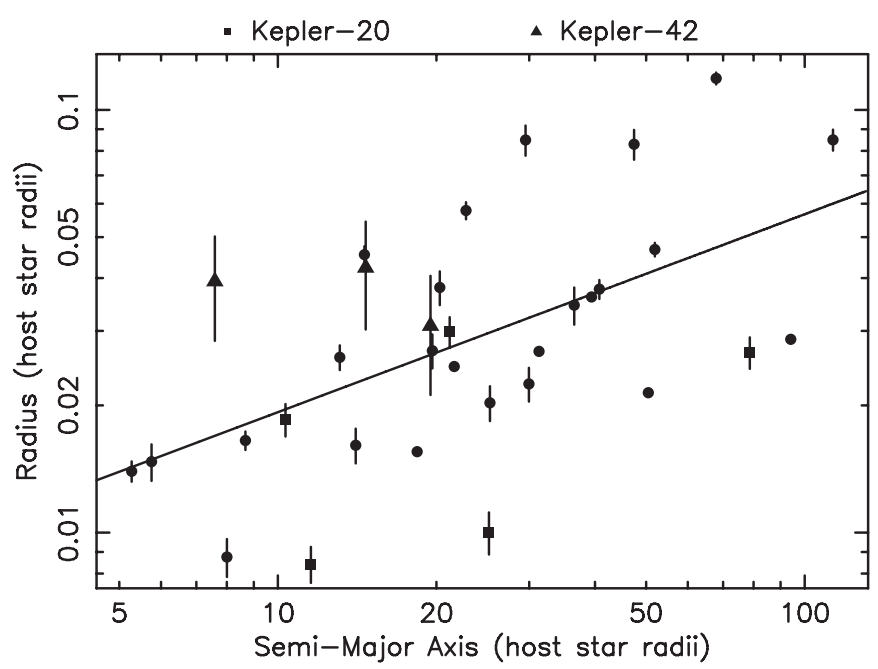

Figure 2. Dependence of planet radius on semimajor axis for the planets in eight of the Kepler multi-planet systems. The values are scaled in units of the radius of the host star. Also shown is a power-law fit to the data.

Stellar masses are generally not well determined for the Kepler stars, but the radii range from $0.17 R_{\odot}$ to $1.82 R_{\odot}$ for Kepler-42 and Kepler-33, respectively. Despite the dramatic differences in the host stars however, there is still a visible upward trend to the radius relationship as a function of semimajor axis. The power-law fit to the data is once again of the form $y=c_{1} x^{c_{2}}$, where $c_{1}=0.00656$ and $c_{2}=0.46814$. Though still positive, the slope of the relation is substantially different from that of the solar system moons. We discuss this in more detail in Section 6.

The lack of data points in the top left of Figure 2 is likely due to a real dearth of planets in that region of parameter space, since large planets at short orbital radii are relatively easy to detect. However, it is worth exploring if the lack of planets in the bottom right is due to Kepler incompleteness in that region since small planets with larger orbital radii are more challenging to find. The probability of a transit occurring is dominated by the radius of the star rather than the radius of the planet (Kane \& von Braun 2008), so the radius of the planet has a minor influence on the probability detection bias. The aspect of Kepler completeness is a complicated issue to quantify since it depends on the intricacies of the planet detection algorithm with respect to correlated noise in the data. The Kepler candidates released by Batalha et al. (2013) show that transiting planets within the bottom-right region of Figure 2 would have been detected if they existed for the multi-planet systems considered in this paper. In addition, these multi-planet systems have been studied in far greater detail than the bulk of the Kepler planet candidates, which lends credence to the superior detection completeness of the multi-planet systems considered here. Future releases of Kepler multi-planet systems will provide further understanding of this completeness as the time baseline of the Kepler photometry increases.

\section{STATISTICAL ANALYSIS}

Given the amount of scatter around the power-law fits to the data shown in Section 2 (Figure 1) and in Section 3 (Figure 2), we include here statistical tests to investigate the strength of the apparent correlations. A robust method to test the correlation lies in the use of the Spearman's rank correlation coefficient. This delivers a value that lies in the range $-1<r_{s}<1$, and a
Table 2

Kepler Planet Separations and Radii

\begin{tabular}{|c|c|c|c|c|}
\hline Planet & $\begin{array}{c}a \\
(\mathrm{AU})\end{array}$ & $\begin{array}{c}R_{p} \\
\left(R_{\oplus}\right)\end{array}$ & \multicolumn{2}{|c|}{ (Host Star Radii) } \\
\hline \multicolumn{5}{|c|}{ Kepler-9 } \\
\hline $\mathrm{b}$ & 0.140 & 9.448 & 29.523 & 0.085 \\
\hline $\mathrm{c}$ & 0.225 & 9.235 & 47.447 & 0.083 \\
\hline $\mathrm{d}$ & 0.027 & 1.638 & 5.757 & 0.015 \\
\hline \multicolumn{5}{|c|}{ Kepler-11 } \\
\hline $\mathrm{b}$ & 0.091 & 1.807 & 18.379 & 0.016 \\
\hline $\mathrm{c}$ & 0.107 & 2.873 & 21.610 & 0.025 \\
\hline $\mathrm{d}$ & 0.155 & 3.120 & 31.305 & 0.027 \\
\hline $\mathrm{e}$ & 0.195 & 4.197 & 39.383 & 0.036 \\
\hline $\mathrm{f}$ & 0.250 & 2.491 & 50.492 & 0.021 \\
\hline $\mathrm{g}$ & 0.466 & 3.333 & 94.116 & 0.029 \\
\hline \multicolumn{5}{|c|}{ Kepler-18 } \\
\hline $\mathrm{b}$ & 0.045 & 1.997 & 8.678 & 0.017 \\
\hline $\mathrm{c}$ & 0.075 & 5.499 & 14.598 & 0.045 \\
\hline $\mathrm{d}$ & 0.117 & 6.991 & 22.752 & 0.058 \\
\hline \multicolumn{5}{|c|}{ Kepler-20 } \\
\hline $\mathrm{b}$ & 0.045 & 1.908 & 10.338 & 0.019 \\
\hline $\mathrm{c}$ & 0.093 & 3.075 & 21.190 & 0.030 \\
\hline $\mathrm{d}$ & 0.345 & 2.749 & 78.678 & 0.027 \\
\hline $\mathrm{e}$ & 0.051 & 0.869 & 11.552 & 0.008 \\
\hline $\mathrm{f}$ & 0.110 & 1.032 & 25.155 & 0.010 \\
\hline \multicolumn{5}{|c|}{ Kepler-30 } \\
\hline $\mathrm{b}$ & 0.180 & 3.905 & 40.755 & 0.038 \\
\hline $\mathrm{c}$ & 0.300 & 12.310 & 67.924 & 0.119 \\
\hline $\mathrm{d}$ & 0.500 & 8.809 & 113.207 & 0.085 \\
\hline \multicolumn{5}{|c|}{ Kepler-32 } \\
\hline $\mathrm{b}$ & 0.050 & 2.199 & 20.292 & 0.038 \\
\hline $\mathrm{c}$ & 0.090 & 1.997 & 36.525 & 0.035 \\
\hline $\mathrm{d}$ & 0.128 & 2.704 & 51.947 & 0.047 \\
\hline $\mathrm{e}$ & 0.032 & 1.504 & 13.109 & 0.026 \\
\hline $\mathrm{f}$ & 0.013 & 0.808 & 5.276 & 0.014 \\
\hline \multicolumn{5}{|c|}{ Kepler-33 } \\
\hline $\mathrm{b}$ & 0.068 & 1.739 & 8.001 & 0.009 \\
\hline $\mathrm{c}$ & 0.119 & 3.198 & 14.052 & 0.016 \\
\hline $\mathrm{d}$ & 0.166 & 5.353 & 19.642 & 0.027 \\
\hline $\mathrm{e}$ & 0.214 & 4.029 & 25.268 & 0.020 \\
\hline $\mathrm{f}$ & 0.254 & 4.465 & 29.960 & 0.022 \\
\hline \multicolumn{5}{|c|}{ Kepler-42 } \\
\hline $\mathrm{b}$ & 0.012 & 0.786 & 14.677 & 0.042 \\
\hline $\mathrm{c}$ & 0.006 & 0.729 & 7.592 & 0.039 \\
\hline $\mathrm{d}$ & 0.015 & 0.572 & 19.485 & 0.031 \\
\hline
\end{tabular}

corresponding probability of the null hypothesis (the hypothesis that the two quantities are not correlated).

Since the data for both the moons and the Kepler planets are best fit by a power law, we convert the data to a linear scale using the logarithm of the values. For the 15 moons that are shown in the right panel of Figure 1, the correlation coefficient is $r_{s}=0.84$, which means that the data exhibit a strong positive correlation. The probability that there is no correlation between the scaled radii and separations is $0.16 \%$. For the 33 Kepler planets shown in Figure 2, the correlation coefficient is $r_{s}=0.53$. This is a positive correlation with a probability of no correlation of $0.29 \%$. Even though the scatter in the plot of the Kepler planets is larger than that for the moons, the probabilities of the null hypothesis are similar to each other due to the additional degrees of freedom for the Kepler sample.

To further test the robustness of this result, we constructed a Monte Carlo simulation that performs a Fisher-Yates shuffle on the Kepler data that randomizes the order of the data values. This was executed 1000 times and in each case the Spearman's 

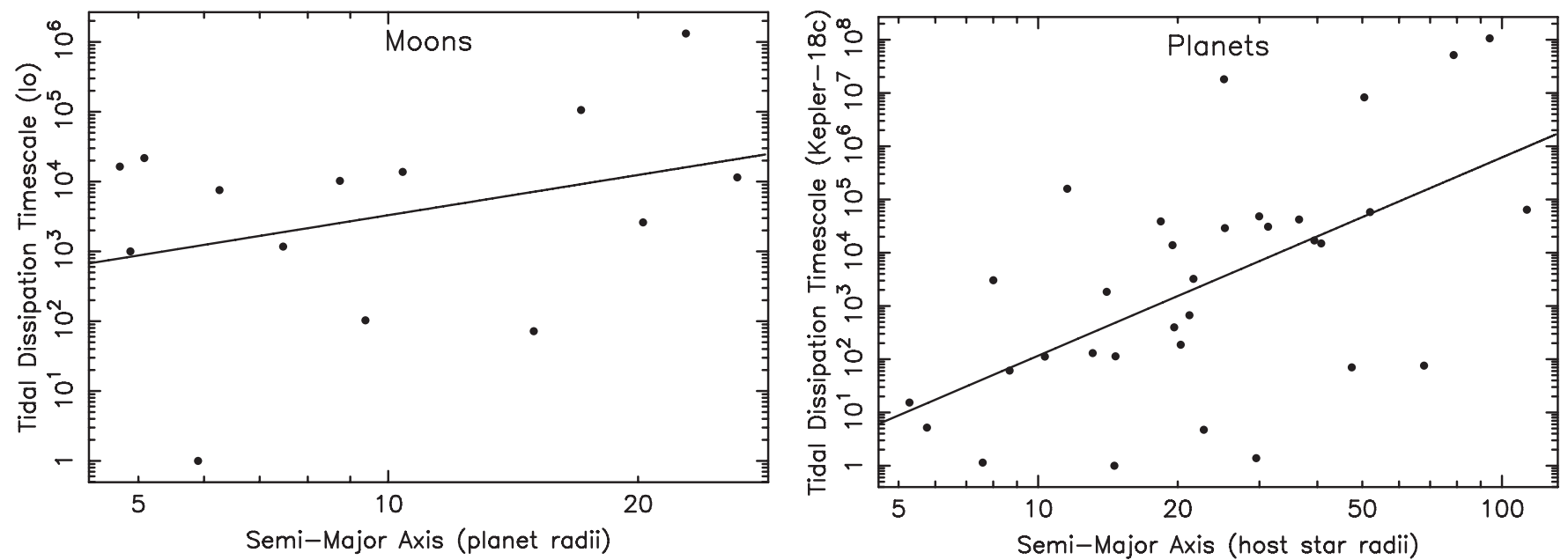

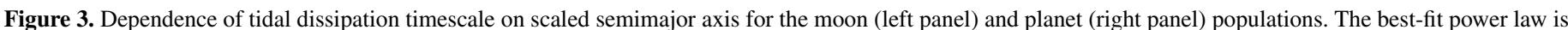
shown in each case.

rank correlation coefficient and corresponding probability were recalculated. The results of this simulation showed that a random ordering of the Kepler scaled radius values has a mean probability of $50 \%$, much higher than that described above. We thus conclude that the correlations shown are statistically significant.

\section{TIDAL DISSIPATION TIMESCALES}

As a further comparison of the moon and planet populations described in previous sections, we investigate the tidal properties of these populations. We use the tidal dissipation timescale in the context of the constant time-lag model (Hut 1981; Eggleton et al. 1998; Leconte et al. 2010), defined as

$$
T_{p}=\frac{1}{9} \frac{M_{p}}{M_{\star}\left(M_{p}+M_{\star}\right)} \frac{a^{8}}{R_{p}^{10}} \frac{1}{\sigma_{p}},
$$

where $M_{p}$ and $R_{p}$ are the mass and radius of the planet, respectively, $M_{\star}$ is the mass of the star, $a$ is the semimajor axis, and $\sigma_{p}$ is the dissipation factor of the planet (Hansen 2010; Bolmont et al. 2011, 2013). We estimate the masses of the planets using the mass-radius relationships of Weiss et al. (2013). We have calculated these timescales for each of the objects in the populations shown in Tables 1 and 2. For the moon population, the parameters for the star and planet in Equation (1) are replaced with the parameters of the planet and moon, respectively. For each population, we express the dissipation timescales in units of the object experiencing the highest tidal effects and thus the lowest tidal dissipation timescale. This object is Io and Kepler-18c for the moons and Kepler planets, respectively.

The results of these calculations are shown in Figure 3 for the moon (left panel) and planet (right panel) populations as a function of their scaled semimajor axes. As was done in Sections 2 and 3, we fit a power law to each distribution of tidal dissipation timescales of the form $y=c_{1} x^{c_{2}}$. For the moons, $c_{1}=0.022$ and $c_{2}=3.723$. For the planets, $c_{1}=40.152$ and $c_{2}=1.915$. In this case there is a notable difference between the two populations in that the slope of the relation between the tidal dissipation timescale and scaled semimajor axis for the planets is approximately twice that for the moons.

Are the differences in tidal timescale distributions of the moons and planets a result of our assumption of a constant $\sigma_{p}$ ?
If $\sigma_{p}$ is arbitrarily multiplied by 1000 then the two distributions are more compatible. There is considerable uncertainty in the dissipation rates of even the best-studied moons, and orders of magnitude uncertainty for exoplanets. It is reasonable to think that, given their larger radii, the dissipation rates of planets should be higher than for moons and their dissipation timescales correspondingly shorter. However, even if we assume a constant fixed $\sigma_{p}$ value for each population, the distributions of the moons and planets are fit by different slopes. The origin and significance of this difference is unclear.

\section{FORMATION SCENARIOS}

A summary of all the solar system moons and Kepler planets discussed in this paper are shown in Figure 4. The radii are all scaled in units of the host, which is a giant planet in the case of the moons and a star in the case of the Kepler planets. Similarly, the separations of the objects from their host are scaled in units of the host radii. Two particular bodies stand out in proportionality to the host: the largest moon (Triton) and the largest planet (Kepler-30c). It is clear from Figure 4 that the different slope of the power-law fits to the data in Sections 2 and 3 is due to the more diverse range of scaled semimajor axes of the Kepler planets rather than any significant difference in scaled radii of the moons/planets. The mean of the scaled semimajor axes and radii for the moons are 11.5 and 0.048 host radii, respectively. For the Kepler planets, the mean of the scaled semimajor axes and radii are 30.9 and 0.035 host radii, respectively. By comparison, the scaled semimajor axes and radii of the Earth's moon are 60.3 and 0.273 , respectively: significantly removed from the objects in this study and almost certainly a result of the very different formation mechanisms involved.

Previous studies have found a host star metallicity correlation with the presence of giant planets (Fischer \& Valenti 2005; Ghezzi et al. 2010). Analysis of Kepler host stars has found that there is no significant correlation of host star metallicity with the abundance of small exoplanets (Buchhave et al. 2012). A similar analysis on the abundance of chemical elements in RV host stars also found that the metallicity is not related to the presence of terrestrial exoplanets (González Hernández et al. 2010). Ramírez et al. (2010) further showed the lack of metallicity correlation in the terrestrial planet hosts by looking specifically at close solar analogs. Thus, the composition of 


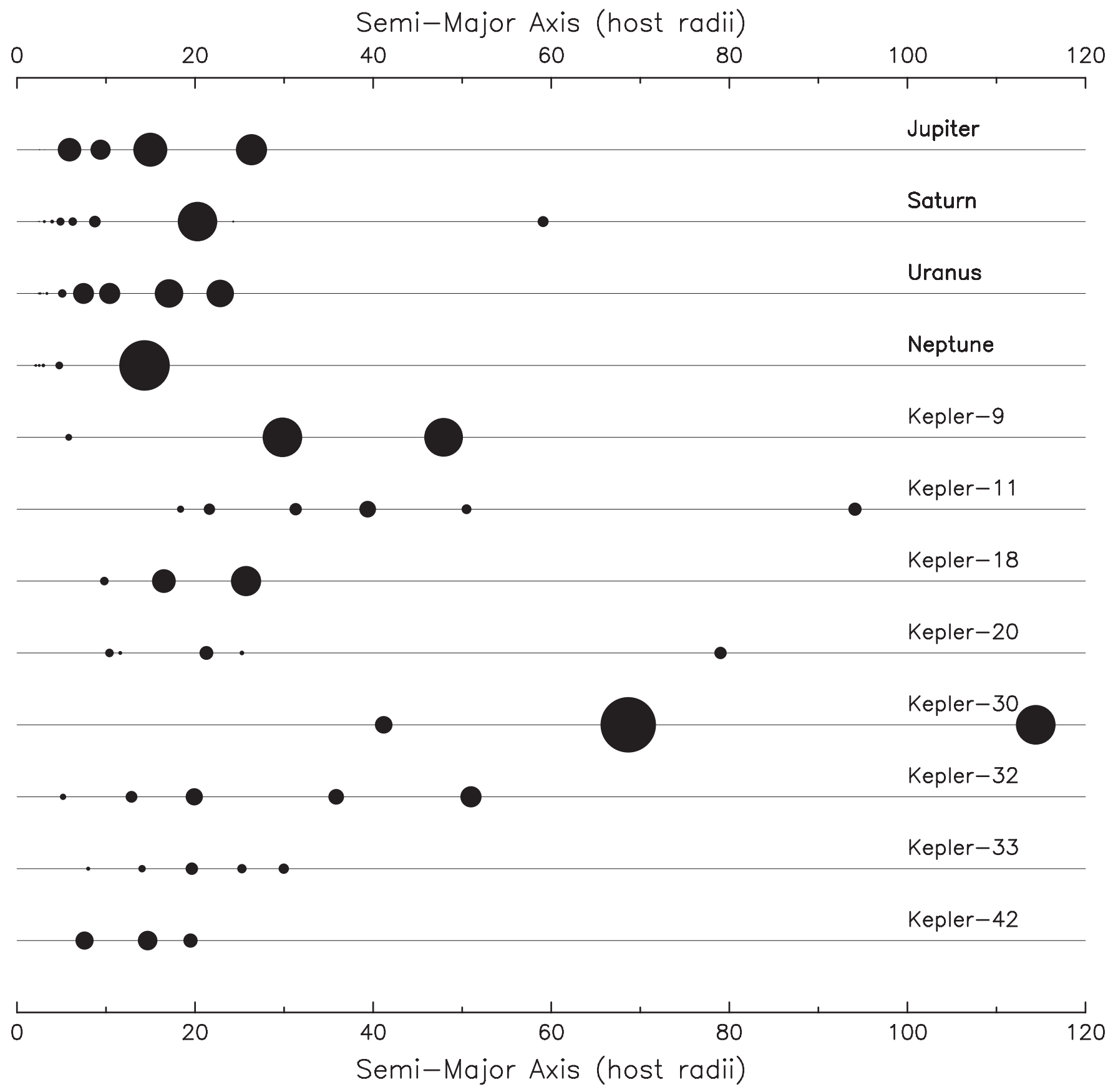

Figure 4. Visualization of the radii and semimajor axes of the solar system moons and Kepler planets discussed in this paper. The radii are all scaled in units of the host, whether the host is a planet or a star. Similarly, the separations of the objects from their host are scaled in units of the host radii. In proportionality to the host, the largest moon is Triton and the largest planet is Kepler-30c.

the host is unlikely to be an indicator of the resulting system properties.

The differences between the solar system moon and exoplanet populations are likely due to differences in formation processes. The compact exoplanetary systems present a particular problem to formation theories, the various issues of which have been explored by Raymond et al. (2008b). In situ formation requires a very massive close-in disk (Raymond et al. 2008b; Chiang \& Laughlin 2013). However, in situ accretion in such a massive disk would invariably trigger orbital decay due to gas drag and type 1 migration. The inward migration of planetary embryos (Terquem \& Papaloizou 2007) tends to trap planets in chains of mean motion resonances (Cresswell \& Nelson 2008; Ogihara \&
Ida 2009). Resonances can be broken by disk turbulence (Pierens et al. 2011) or on longer timescales by dynamical instabilities (Terquem \& Papaloizou 2007; Matsumoto et al. 2012) or tidal dissipation (Batygin \& Morbidelli 2013). In contrast, Io, Europa, and Ganymede are located in the 4:2:1 Laplace resonance. This is interpreted as strong evidence that the moons underwent inward migration either during formation or soon thereafter (Greenberg 1987; Ogihara \& Ida 2012; Peale \& Lee 2002).

Canup \& Ward (2006) proposed that the maximum size of moons may be limited by the migration process. In their scenario, larger moons migrate faster through the circumplanetary disk and collide with the planet. An alternative proposal by Crida \& Charnoz (2012) suggests that moons may be produced 
by the viscous spreading of massive disks inside the Roche limit analogous to Saturn's rings. In their model, the outwardmigrating portion of the disk - pushed out in part by tidal forcing from the giant planet-naturally coalesces into one or more satellites with characteristic properties.

One aspect of the formation differences between the two populations is the materials available during formation, which can affect the final composition and density of the bodies. A significant role in the formation process in the solar system is played by the "snow line," which is the radial distance from the center of a protostellar disk beyond which water molecules can efficiently condense to form ice (Inaba et al. 2003). Beyond the snow line, planetary accretion events have access to much more material in the form of icy volatiles from which to form substantial cores for rapid gas accretion (Lissauer 1987). Although this concept has largely been developed in the context of the solar system, attempts are being made to apply these ideas to the diverse range of stellar masses and exoplanetary systems (Kennedy et al. 2006; Kennedy \& Kenyon 2008). The regular moons that form in situ around giant planets beyond the snow line may form from similar material to that of the giant planet, resulting in a lower mean density with respect to the terrestrial planets, whose formation material consists of mostly refractory elements (Cassen 2001; Raymond et al. 2009b; Bond et al. 2010). If indeed there is a spatial dependence in the exoplanetary abundances then it will be reflected in the mean densities of the moons of those planets. For example, the mean of the mean densities for the solar system terrestrial planets is $5.0 \mathrm{~g} \mathrm{~cm}^{-3}$. The mean of the mean densities for the Galilean moons is $2.6 \mathrm{~g} \mathrm{~cm}^{-3}$ and is significantly lower as one looks to the moons of Saturn, Uranus, and Neptune. Most of the Kepler confirmed planets have poorly estimated densities since their masses often only have upper limits.

An additional comparison that may be made between the two populations is that of mutual inclinations. The mutual inclinations of the Kepler multi-planet systems have been an emerging topic as more of those systems are discovered, and have been studied in some detail by such authors as Lissauer et al. (2011b), Fang \& Margot (2012), and Tremaine \& Dong (2012). The compact Kepler systems have very small mutual inclinations by virtue of their transit detection, and it was shown by Lissauer et al. (2011b) that the inclination dispersion of these systems generally has a mean $<10^{\circ}$. Of the solar system moons shown in Table 1, only two have inclinations larger than 1.0 with respect to the local Laplace plane. These are Miranda (4.338), which has strong evidence of significant geological, tidal, and orbital evolution (Tittemore \& Wisdom 1990), and Triton (156.8), which, as previously mentioned, was likely captured into its current retrograde orbit. The strong mutual inclination present in the remainder of the major moons is similar to that exhibited by the Kepler compact systems. An equivalent comparison could be made between the eccentricity distributions between the two populations as an indicator of analogous formation mechanisms. However, although the eccentricity distribution of Kepler planets has been investigated from a statistical perspective (Moorhead et al. 2011; Kane et al. 2012), determination of the eccentricity for individual planets from photometry alone is a difficult exercise (Kipping 2008).

A further aspect of the observed orbital configurations of the two populations is the allowed regions of orbital stability. Stable orbits for multi-planet systems are a strong function of the mass of those planets as well as their respective separations, and thus is far more restrictive for planets than it is for less massive moons (Chambers et al. 1996; Raymond et al. 2008a). Such stability analysis is able to determine exclusion zones for the presence of planets in the habitable zone (Kopparapu $\&$ Barnes 2010). Several studies have been undertaken with respect to the stability of compact planetary systems that have found that these compact configurations are not only achievable (Smith \& Lissauer 2009; Funk et al. 2010), but may even be the preferred result of planet formation (Raymond et al. 2009a). Continued discoveries of compact exoplanetary systems will reveal if these configurations are greatly influenced by these stability considerations.

\section{EXTRAPOLATION TO BROWN DWARFS}

This work has focused on two populations, planets orbiting stars and moons orbiting planets. In between these two populations lies a mass regime that includes potential companions to brown dwarfs. If the same trend could be applied to such systems, then one may expect to find sub-Earth size planets in relatively short-period orbits around brown dwarfs. Such planets would have exceptionally high geometric transit probabilities, but a successful transit detection would be hindered by the relative faintness of the brown dwarfs. A recent study by Belu et al. (2013) suggests several prospects for conducting such a survey, including a space-based or longitude-distributed network of ground-based observations. A key advantage in the observational prospects of these systems is that the orbital period for close-in planets is small enough to allow monitoring of a complete orbit to occur within a single night of ground-based observations. However, the expectation that such systems would follow a trend similar to those described in this work depends highly upon their formation mechanisms. Thus, the detection of planets around brown dwarfs would play a key role in providing links between the planet and moon formation scenarios described in Section 6.

\section{CONCLUSIONS}

The planetary detections by the Kepler mission have allowed access to the radii and mean densities for terrestrial planets in multi-planet systems. One of the big surprises from these multi-planet systems is the frequency of planets in compact orbital configurations. The high occurrence implies that compact systems are relatively common and bear the signature of fundamental formation processes that differ significantly from our own solar system. However, we have shown here that there is a correlation between scaled radii and semimajor axes for these planets (despite the large range of stellar properties) and that this correlation bears a strong similarity to the same correlation for the moons of the solar system giant planets. The main difference between the two populations is the gradient of the correlation, which is dominated by the Kepler planets extending to larger scaled orbital separations. The observed correlations may be explained by inward migration of the moons or perhaps a difference in materials present during formation. Thus the different correlation gradients between the two populations are possibly the result of differences in resonance-trapping mechanisms between protomoon disks around planets and protoplanet disks around stars, which in turn are due to differences in relative disk masses and composition. However, since migration occurs for both populations, a difference in disk viscosity and thus turbulent fluctuations may also play a key role in the resulting compactness of the systems. This may be tested from the 
properties of moons that are detected around exoplanets close to their host stars. Searches for exomoons in the Kepler data are being undertaken by Kipping et al. (2012). Although there are no current capabilities to do so, an eventual comparison of the densities between solar system moons and exomoons will be useful to study. If the density of the exomoons differs substantially from the moons of our solar system, then it will shed further light on the formation and migration mechanisms for both the solar system moons and the Kepler compact planetary systems.

The authors would like to thank Jason Eastman, Gregory Laughlin, Philip Muirhead, and Jonathan Swift for insightful discussions. Thanks are also due to the anonymous referee, whose comments improved the quality of the paper. This research has made use of the NASA Exoplanet Archive, which is operated by the California Institute of Technology under contract with the National Aeronautics and Space Administration under the Exoplanet Exploration Program. The authors acknowledge financial support from the National Science Foundation through grant AST-1109662.

\section{REFERENCES}

Agnor, C. B., \& Hamilton, D. P. 2006, Natur, 441, 192

Batalha, N. M., Rowe, J. F., Bryson, S. T., et al. 2013, ApJS, 204, 24 Batygin, K., \& Morbidelli, A. 2013, AJ, 145, 1

Belu, A. R., Selsis, F., Raymond, S. N., et al. 2013, ApJ, 768, 125 Bolmont, E., Raymond, S. N., \& Leconte, J. 2011, A\&A, 535, 94 Bolmont, E., Selsis, F., Raymond, S. N., et al. 2013, A\&A, 556, 17 Bond, J. C., Lauretta, D. S., \& O’Brien, D. P. 2010, Icar, 205, 321 Borucki, W. J., Koch, D. G., Basri, G., et al. 2011a, ApJ, 728, 117 Borucki, W. J., Koch, D. G., Basri, G., et al. 2011b, ApJ, 736, 19 Buchhave, L. A., Latham, D. W., Johansen, A., et al. 2012, Natur, 486, 375 Canup, R. M., \& Ward, W. R. 2006, Natur, 441, 834 Cassen, P. 2001, M\&PS, 36, 671

Chambers, J. E., Wetherill, G. W., \& Boss, A. P. 1996, Icar, 119, 261 Chiang, E., \& Laughlin, G. 2013, MNRAS, 431, 3444

Ciardi, D. R., Fabrycky, D. C., Ford, E. B., et al. 2013, ApJ, 763, 41 Cochran, W. D., Fabrycky, D. C., Torres, G., et al. 2011, ApJS, 197, 7

Colombo, G., Franklin, F. A., \& Shapiro, I. I. 1974, AJ, 79, 61

Cresswell, P., \& Nelson, R. P. 2008, A\&A, 482, 677

Crida, A., \& Charnoz, S. 2012, Sci, 338, 1196

Dawson, R. I., \& Murray-Clay, R. 2012, ApJ, 750, 43

Eggleton, P. P., Kiseleva, L. G., \& Hut, P. 1998, ApJ, 499, 853

Fabrycky, D. C., Ford, E. B., Steffen, J. H., et al. 2012, ApJ, 750, 114

Fang, J., \& Margot, J.-L. 2012, ApJ, 761, 92

Fischer, D. A., \& Valenti, J. 2005, ApJ, 622, 1102

Fressin, F., Torres, G., Rowe, J. F., et al. 2012, Natur, 482, 195

Frouard, J., Vienne, A., \& Fouchard, M. 2011, A\&A, 532, 44

Funk, B., Wuchterl, G., Schwarz, R., Pilat-Lohinger, E., \& Eggl, S. 2010, A\&A, 516,82
Gaspar, H. S., Winter, O. C., \& Vieira Neto, E. 2011, MNRAS, 415, 1999

Gautier, T. N., Charbonneau, D., Rowe, J. F., et al. 2012, ApJ, 749, 15

Ghezzi, L., Cunha, K., Smith, V. V., et al. 2010, ApJ, 720, 1290

González Hernández, J. I., Israelian, G., Santos, N. C., et al. 2010, ApJ, 720,1592

Greenberg, R. 1987, Icar, 70, 334

Hansen, B. M. S. 2010, ApJ, 723, 285

Holman, M. J., Fabrycky, D. C., Ragozzine, D., et al. 2010, Sci, 330, 51

Hut, P. 1981, A\&A, 99, 126

Inaba, S., Wetherill, G. W., \& Ikoma, M. 2003, Icar, 166, 46

Jewitt, D., \& Haghighipour, N. 2007, ARA\&A, 45, 261

Kane, S. R., Ciardi, D. R., Gelino, D. M., \& von Braun, K. 2012, MNRAS, 425,757

Kane, S. R., \& von Braun, K. 2008, ApJ, 689, 492

Kennedy, G. M., \& Kenyon, S. J. 2008, ApJ, 673, 502

Kennedy, G. M., Kenyon, S. J., \& Bromley, B. C. 2006, ApJL, 650, L139

Kipping, D. M. 2008, MNRAS, 389, 1383

Kipping, D. M., Bakos, G. Á., Buchhave, L., Nesvorný, D., \& Schmitt, A. 2012, ApJ, 750, 115

Kopparapu, R. K., \& Barnes, R. 2010, ApJ, 716, 1336

Leconte, J., Chabrier, G., Baraffe, I., \& Levrard, B. 2010, A\&A, 516, 64

Levison, H. F., Morbidelli, A., Van Laerhoven, C., Gomes, R., \& Tsiganis, K. 2008, Icar, 196, 258

Lissauer, J. J. 1987, Icar, 69, 249

Lissauer, J. J., Fabrycky, D. C., Ford, E. B., et al. 2011a, Natur, 470, 53

Lissauer, J. J., Jontof-Hutter, D., Rowe, J. F., et al. 2013, ApJ, 770, 131

Lissauer, J. J., Marcy, G. W., Rowe, J. F., et al. 2012, ApJ, 750, 112

Lissauer, J. J., Ragozzine, D., Fabrycky, D. C., et al. 2011b, ApJS, 197, 8

Lovis, C., Ségransan, D., Mayor, M., et al. 2011, A\&A, 528, 112

Matsumoto, Y., Nagasawa, M., \& Ida, S. 2012, Icar, 221, 624

Moorhead, A. V., Ford, E. B., Morehead, R. C., et al. 2011, ApJS, 197, 1

Muirhead, P. S., Johnson, J. A., Apps, K., et al. 2012, ApJ, 747, 144

Ogihara, M., \& Ida, S. 2009, ApJ, 699, 824

Ogihara, M., \& Ida, S. 2012, ApJ, 753, 60

Peale, S. J. 1978, Icar, 36, 240

Peale, S. J., \& Lee, M. H. 2002, Sci, 298, 593

Pierens, A., Baruteau, C., \& Hersant, F. 2011, A\&A, 531, 5

Ramírez, I., Asplund, M., Baumann, P., Meléndez, J., \& Bensby, T. 2010, A\&A, 521,33

Raymond, S. N., Barnes, R., \& Gorelick, N. 2008a, ApJ, 689, 478

Raymond, S. N., Barnes, R., \& Mandell, A. M. 2008b, MNRAS, 384, 663

Raymond, S. N., Barnes, R., Veras, D., et al. 2009a, ApJL, 696, L98

Raymond, S. N., O'Brien, D. P., Morbidelli, A., \& Kaib, N. A. 2009b, Icar, 203, 644

Sanchis-Ojeda, R., Fabrycky, D. C., Winn, J. N., et al. 2012, Natur, 487, 449

Sheppard, S. S., \& Jewitt, D. C. 2003, Natur, 423, 261

Sheppard, S. S., Jewitt, D., \& Kleyna, J. 2005, AJ, 129, 518

Sheppard, S. S., Jewitt, D., \& Kleyna, J. 2006, AJ, 132, 171

Smith, A. W., \& Lissauer, J. J. 2009, Icar, 201, 381

Swift, J. J., Johnson, J. A., Morton, T. D., et al. 2013, ApJ, 764, 105

Terquem, C., \& Papaloizou, J. C. B. 2007, ApJ, 654, 1110

Tittemore, W. C., \& Wisdom, J. 1990, Icar, 85, 394

Torres, G., Fressin, F., Batalha, N. M., et al. 2011, ApJ, 727, 24

Tremaine, S., \& Dong, S. 2012, AJ, 143, 94

Tuomi, M. 2012, A\&A, 543, 52

Weiss, L. M., Marcy, G. W., Rowe, J. F., et al. 2013, ApJ, 768, 14 